\title{
MÉTODOS DE ESTIMAÇÃO DE ALTURA DE ÁRVORES EM FLORESTA ESTACIONAL SEMIDECIDUAL
}

\author{
Rafaella De Angeli Curto ${ }^{1}$, Gilson Fernandes da $\mathrm{Silva}^{2}$, Carlos Pedro Boechat Soares ${ }^{3}$, \\ Leandro Tose Martins ${ }^{4}$, Hassan Camil David ${ }^{5}$ \\ ${ }^{1}$ Eng ${ }^{\mathrm{a}}$ Florestal, Doutoranda em Engenharia Florestal, UFPR, Curitiba, PR, Brasil - rafaellacurto@ yahoo.com.br \\ ${ }^{2}$ Eng. Florestal, Dr., Depto. de Engenharia Florestal, UFES, Jerônimo Monteiro, ES, Brasil - gilson.silva@ @q.cnpq.br \\ ${ }^{3}$ Eng. Florestal, Dr., Depto. de Engenharia Florestal, UFV, Viçosa, MG, Brasil - csoares@ufv.br \\ ${ }^{4}$ Eng. Florestal, M.Sc., Jerônimo Monteiro, ES, Brasil - ltosemartins@ @otmail.com \\ ${ }^{5}$ Eng. Florestal, Mestrando em Engenharia Florestal, UFPR, Curitiba, PR, Brasil - hassancamil@ hotmail.com
}

Recebido para publicação: 02/05/2012 - Aceito para publicação: 23/01/2013

\begin{abstract}
Resumo
O estudo foi realizado na Floresta do Rosal, Guaçuí, ES, para avaliar precisão e tempo de execução de métodos de estimação de altura, em diferentes condições de terreno, com os métodos hipsômetro Vertex (1); clinômetro digital (2); estimação com auxílio de uma régua de 15 metros (3); e estimações visuais com treinamento (4) e sem treinamento (5), em três classes de altura: 1 (15,00-17,99 m); 2 (18,00$20,99 \mathrm{~m})$; e 3 (> 21,00 m). Foram mensurados 124 indivíduos em terreno plano e 87 em terreno inclinado. Os dados de altura foram comparados pelo teste $t$, análises gráficas de resíduos e estatísticas complementares. Para avaliar o tempo, foi realizado teste $t$. O método 5 apresentou o pior desempenho quanto à precisão, enquanto que o melhor desempenho foi do método 4. A declividade afetou negativamente o desempenho dos equipamentos digitais e favoreceu o método 3 . Houve tendência em subestimar altura com o aumento das classes. Os métodos 4 e 5 consumiram menos tempo e o 3 , o maior tempo médio. $\mathrm{O}$ efeito da classe no tempo médio em terreno plano foi igual para todos os métodos, à exceção do 2. Em terreno inclinado, os métodos 3 e 4 não tiveram desempenhos alterados.

Palavras-chave: Altura total; precisão; tempo.
\end{abstract}

\begin{abstract}
Methods of estimation of height of trees in Semideciduous Seasonal Forest. The research was conducted in Forest of Rosal, Guaçuí-ES, in order to evaluate accuracy and running time of five height estimating methods in different ground conditions; the methods are as follow: Vertex hypsometer (1); Digital Clinometer (2), estimation with aid of a 15 meters ruler (3), and visual estimation with training (4) and untrained (5) in three height classes: $1(15.00-17.99 \mathrm{~m}), 2(18.00$ $20.99 \mathrm{~m})$, and $3(>21.00 \mathrm{~m})$. It were measured 124 individuals in flat ground and 87 individuals in sloping ground. The height data were compared by t test and residual graphic analysis and additional statistics. To evaluate time, it was improved a $t$ test. The method 5 had the worst performance in terms of accuracy, the best performance was improved by the method 4 . The slop affected negatively the digital equipment performance and favored the method 3. There was a tendency to underestimate height as classes increase. The methods 4 and 5 consumed less time and method 3 consumed the highest average time. The class effect on average time on flat ground was the same for all methods but method 2. On slope ground, performance of methods 3 and 4 weren't altered.

Keywords: Height; accuracy; time.
\end{abstract}

\section{INTRODUÇÃO}

Para a realização do manejo florestal, visando à produção sustentável, é de fundamental importância conhecer as características da floresta, as quais podem ser obtidas por inventário florestal.

De acordo com Husch et al. (1993), os inventários florestais são procedimentos para obtenção de informações sobre os recursos florestais e de características das áreas sobre as quais estão crescendo, sendo as variáveis mais utilizadas a altura e o diâmetro.

Estimar com precisão a altura das árvores em florestas nativas é uma operação mais difícil do que em povoamentos plantados, especialmente para as árvores mais altas. Isso ocorre porque os aparelhos 
mais utilizados para a estimação da altura se baseiam em princípios trigonométricos, sendo necessário que o mensurador se localize a uma distância conhecida da árvore.

Em alguns aparelhos, a distância é predeterminada; em outros, como os hipsômetros a laser ou de ondas de ultrassom, há dispositivos eletrônicos que agilizam as leituras, dispensando o uso de trena, permitindo que o mensurador fique a cada leitura em diferentes distâncias, possibilitando maior flexibilidade (CAMPOS; LEITE, 2009).

Por outro lado, o mensurador, às vezes, é obrigado a ficar bem próximo da árvore, pois, caso se afaste, provavelmente perderá a visão da copa, impedindo a leitura. Ao ficar próximo da árvore, o mensurador realiza uma inclinação maior do aparelho, aumentando as chances de leituras equivocadas.

Diante da importância e dificuldades apresentadas para a estimação de altura de árvores em florestas nativas, o objetivo foi avaliar a precisão e o desempenho dos métodos em termos de tempo de execução, em diferentes condições do terreno.

\section{METODOLOGIA}

Este trabalho foi desenvolvido em um fragmento de Floresta Estacional Semidecidual, conhecida como Floresta do Rosal, localizada no município de Guaçuí, ES, com coordenadas geográficas de acesso de $20^{\circ} 53^{\prime}$ latitude sul e $41^{\circ} 42^{\prime}$ ' de longitude oeste.

Para avaliar diferentes métodos de estimação de altura em diferentes condições do terreno, foram propostos cinco métodos, divididos em três classes de altura (Tabela 1). Para comparar os tratamentos definidos, foram selecionados e mensurados 87 indivíduos localizados em terreno com declividade superior a 5 graus, considerado neste estudo como terreno inclinado, e 124 indivíduos em terreno com declividade abaixo desta, considerado como terreno plano, admitindo-se que a declividade pode ter influência no desempenho dos métodos. $\mathrm{O}$ total de árvores apresenta-se distribuído nas três diferentes classes de altura (1, 2 e 3), conforme tabela 1.

Tabela 1. Tratamentos avaliados na estimação de altura total, considerando cinco métodos em três classes de altura, e a distribuição do número de árvores por classe de altura e tipo de terreno.

Table 1. Treatments evaluated in the estimation of total height, considering five methods in three height classes, and distribution of number of trees per height class and groung types.

\begin{tabular}{lccccc}
\hline \multirow{2}{*}{ Tratamento } & \multirow{2}{*}{ Método de estimação } & \multirow{2}{*}{ Classe de altura $(\mathbf{m})$} & \multicolumn{2}{c}{ Número de árvores/terreno } & \\
\cline { 4 - 5 } & & & Plano & Inclinado & Total \\
\hline 1 & 1 - Hipsômetro Vertex & $1-15,00-17,99$ & 51 & 40 & 91 \\
2 & 1 - Hipsômetro Vertex & $2-18,00-20,99$ & 36 & 21 & 57 \\
3 & 1 - Hipsômetro Vertex & $3->21,00$ & 37 & 26 & 63 \\
\hline & & & 124 & 87 & 211 \\
\hline Total & 2 - Clinômetro Digital & $1-15,00-17,99$ & 51 & 40 & 91 \\
5 & 2 - Clinômetro Digital & $2-18,00-20,99$ & 36 & 21 & 57 \\
6 & 2 - Clinômetro Digital & $3->21,00$ & 37 & 26 & 63 \\
\hline Total & & & 124 & 87 & 211 \\
\hline 7 & 3 - Auxílio de régua (15m) & $1-15,00-17,99$ & 51 & 40 & 91 \\
8 & 3 - Auxílio de régua (15m) & $2-18,00-20,99$ & 36 & 21 & 57 \\
9 & 3 - Auxílio de régua (15m) & $3->21,00$ & 37 & 26 & 63 \\
\hline Total & & & 124 & 87 & 211 \\
\hline 10 & 4 - Visual com reinamento & $1-15,00-17,99$ & 51 & 40 & 91 \\
11 & 4 - Visual com reinamento & $2-18,00-20,99$ & 36 & 21 & 57 \\
12 & 4 - Visual com reinamento & $3->21,00$ & 37 & 26 & 63 \\
\hline Total & & & 124 & 87 & 211 \\
\hline 13 & 5 - Visual sem treinamento & $1-15,00-17,99$ & 51 & 40 & 91 \\
14 & 5 - Visual sem treinamento & $2-18,00-20,99$ & 36 & 21 & 57 \\
15 & 5 - Visual sem treinamento & $3->21,00$ & 37 & 26 & 63 \\
\hline Total & & & 124 & 87 & 211 \\
\hline
\end{tabular}

Para a estimação da altura total pelo método 1, foi utilizado o hipsômetro VertexIII (Código VertexIII-360, incluindo o instrumento de medição, transponder, adaptador e suporte), o qual, antes de se iniciarem as atividades, foi devidamente calibrado. 
Para efetuar as estimações, o transponder era fixado a uma altura pré-definida da árvore (1,30 metros do solo). A partir daí, era possível obter a distância do mensurador até a árvore de forma automática (podendo o mensurador se posicionar em diferentes distâncias da mesma), além da obtenção de até seis leituras consecutivas de altura total em cada árvore, sendo que a média dessas leituras foi considerada como a altura total.

Sucessivos testes mostraram que o equipamento usado neste trabalho não efetua correção de inclinações. Assim, para terrenos inclinados, foi realizada a medição do ângulo $(\theta)$ em graus, em cada situação, a fim de efetuar as devidas correções, aplicando-se uma relação de cosseno conforme apresentado por Soares et al. (2006), em que $\mathrm{D}_{\text {campo }}=$ distância medida no campo e $\mathrm{L}=$ distância no plano horizontal. Assim:

$$
D_{\text {campo }}=L / \cos \theta
$$

Para a estimação com o método 2, clinômetro digital (clinômetro eletrônico Haglof), as estimativas também puderam ser feitas a qualquer distância da árvore, porém essa distância foi obtida com o uso de uma trena. Uma vez conhecida a que distância o mensurador estava posicionado, foi possível realizar a estimativa da altura total de forma automática. Para terrenos inclinados, houve necessidade de medição do ângulo de declividade do terreno, para a correção da declividade, tal como utilizado para o Vertex.

Os métodos 1 e 2 foram aplicados simultaneamente, para que, no caso de terrenos inclinados, obtendo-se apenas uma medida da declividade do terreno, fosse possível a comparação da precisão dos dois equipamentos.

Para a execução dos métodos 4 e 5 , a estimação da altura total da primeira árvore foi realizada por dois operadores por meio de avaliação visual. Na sequência, para a estimação da altura total das próximas árvores, um dos operadores passou a receber treinamento, ou seja, ele era informado sobre a medida real da árvore anterior, obtida por escalada, de modo que pudesse balizar a sua estimativa para as árvores seguintes (Método 4), e o outro operador continuou a estimar a altura sem qualquer balizamento (Método 5).

Em um segundo momento, o operador que realizou o método 4 realizou também a estimação visual com o auxílio de uma régua graduada de 15 metros (Método 3), em que a régua era apoiada à árvore, visando criar uma referência para o operador. Esses dois métodos foram aplicados pelo mesmo operador, visando avaliar se, com o passar do tempo, as referências obtidas pelo operador com a execução do método 4 foram perdidas.

\section{Análise da precisão dos métodos aplicados}

Uma vez obtidas as alturas totais por meio de escalada e pelos métodos propostos, foram aplicados testes de médias, no caso o teste $t$ para amostras independentes, a 5\% de probabilidade, para comparar as alturas médias obtidas pelos métodos e os erros médios cometidos para cada método de estimação. Foram realizadas também análises gráficas de resíduos, com o intuito de se verificar eventuais tendências na obtenção das alturas, e testes complementares à análise gráfica dos resíduos. Os valores residuais utilizados na construção dos gráficos (erro\%) e os critérios e respectivos estimadores dos testes complementares encontram-se na tabela 2.

Com base na análise das estatísticas $V, M D$ e $D P D$, procedeu-se à ordenação das funções segundo o maior ou menor grau de precisão, sendo atribuídos pesos de 1 a 5 , de acordo com os resultados das estatísticas. Foi considerado o método (tratamento) mais preciso aquele que resultou em menor somatório nas notas.

\section{Análise do tempo médio para os métodos aplicados}

Para avaliar a velocidade de execução dos métodos, efetuou-se a marcação do tempo necessário para estimar a altura total de cada indivíduo, utilizando-se um cronômetro.

Para avaliar o tempo de execução do método 1, foi considerado o ato de colocar o transponder na base da árvore, afastar-se dela, escolhendo um ponto de possível visualização da copa da árvore e transponder, e realizar a estimativa da altura.

Para o método 2, avaliou-se o tempo necessário para se afastar da árvore, escolhendo um ponto de possível visualização da copa e base da mesma, esticar a trena e realizar a estimativa de altura. Para 
terrenos inclinados, tanto para o método 1 quanto para o 2, acrescentou-se o tempo para a medição do ângulo de declividade do terreno.

Tabela 2. Critérios e respectivos estimadores para avaliação dos métodos de estimação de altura total.

Table 2. Criteria and respective estimators for evaluation of height estimating methods.

\begin{tabular}{lc}
\hline Critérios & Estimadores \\
Erro $(\%):$ & Erro $(\%)=\frac{Y_{i}-\hat{Y_{i}}}{Y_{i}} 100$ \\
Viés $(V):$ & $V=\frac{\sum_{i=1}^{n} Y i-\sum_{i=1}^{n} Y i}{n}$ \\
Média das diferenças absolutas $(M D):$ & $M D=\frac{\sum_{i=1}^{n} / Y i-Y i /}{n}$ \\
Desvio padrão das diferenças $(D P D):$ & $\sqrt{\left(\sum_{i=1}^{n} d i^{2}-\left(\sum_{i=1}^{n} d i\right)^{2} / n\right)}$ \\
\hline
\end{tabular}

$Y i$ : altura observada obtida pela escalada; $Y i$ :altura estimada pelos cinco métodos propostos; $n$ número de observações; e $d i=(Y i-\hat{Y} i)$.

Para o método 3, levou-se em conta o tempo necessário para abrir toda a régua, visualizar a copa, realizar a estimativa de altura e fechar a régua.

Para a execução dos métodos 4 e 5 , foi cronometrado o tempo necessário para visualizar a copa e realizar a estimativa de altura, lembrando que, para o método 4, o mensurador recebeu informação da altura total do último indivíduo avaliado.

Uma vez realizada a medição do tempo para os cinco métodos propostos, foram aplicados testes de médias, no caso o teste $t$ para amostras independentes, a 5\% de probabilidade, para comparar o tempo médio obtido.

\section{RESULTADOS E DISCUSSÃO}

\section{Análise da precisão dos métodos aplicados}

Teste de média para as alturas estimadas em cada método e para o erro cometido

Na tabela 3 estão apresentados os testes de médias para os tratamentos avaliados, para terreno plano e inclinado, considerando-se a estatística $t$ para amostras independentes, a $5 \%$ de probabilidade. Admitindo que a hipótese estatística é de que os cinco métodos avaliados têm o mesmo desempenho, em termos de precisão, nas diferentes classes, não deveria haver diferença estatística entre os seguintes tratamentos:

Hipótese para classe 1) $\mathrm{T}_{1}=\mathrm{T}_{4}=\mathrm{T}_{7}=\mathrm{T}_{10}=\mathrm{T}_{13}$

Hipótese para classe 2) $\mathrm{T}_{2}=\mathrm{T}_{5}=\mathrm{T}_{8}=\mathrm{T}_{11}=\mathrm{T}_{14}$

Hipótese para classe 3) $\quad \mathrm{T}_{3}=\mathrm{T}_{6}=\mathrm{T}_{9}=\mathrm{T}_{12}=\mathrm{T}_{15}$

Entretanto, com base na tabela 3, para terreno plano e levando-se em conta a altura média, os tratamentos T7 e T13 (estimação com auxílio da régua de 15 metros e estimação sem treinamento) diferiram dos tratamentos T1 e T4 na classe1; nas classes 2 e 3, porém, apenas os tratamentos T14 e T15 (método sem treinamento), respectivamente, diferiram dos demais. $\mathrm{O}$ mesmo resultado foi encontrado quando se avaliou a variável erro médio, dando indicações iniciais de que o método de estimar a altura da árvore sem treinamento teve o pior desempenho, como se esperava. Por outro lado, não se esperava que o método em que empregou a régua como auxílio fosse diferente dos demais, sobretudo na primeira classe, por se tratar de árvores mais baixas. 
Para o terreno inclinado, avaliando a altura média, foram encontrados resultados semelhantes aos obtidos no terreno plano, com ressalva para a classe 1, em que o método que empregou o Vertex (T1) passou, em geral, a diferir dos demais. Quando se avalia o erro médio, nota-se que, na classe 1, a declividade afetou negativamente o desempenho dos equipamentos digitais, especialmente do Vertex (T1). Para as classes 2 e 3, manteve-se a tendência de mesmo desempenho dos métodos, com exceção do método sem treinamento, o qual manteve o pior desempenho em terreno inclinado, para todas as classes.

Tabela 3. Comparação da precisão entre os tratamentos avaliados nas diferentes classes de altura, tal como definido na tabela 1 , em terreno plano e inclinado, considerando-se as variáveis altura média e erro relativo.

Table 3. Comparison of accuracy among the treatments evaluated in different height classes, as defined in table 1, on flat ground and sloping ground, considering the variables height and relative error.

\begin{tabular}{|c|c|c|c|c|c|c|c|c|c|c|c|}
\hline \multicolumn{6}{|c|}{ Estimativa da altura da árvore - Terrenos planos } & \multicolumn{6}{|c|}{ Estimativa da altura da árvore - Terrenos inclinados } \\
\hline $\mathrm{T}_{1}=16,62$ & (A) a & $\mathrm{T}_{2}=18,38$ & (A) $\mathrm{b}$ & $\mathrm{T}_{3}=21,93$ & (A) $\mathrm{c}$ & $\mathrm{T}_{1}=15,15$ & (A) $\mathrm{a}$ & $\mathrm{T}_{2}=18,53$ & (A) $b$ & $\mathrm{~T}_{3}=21,77$ & (A) $\mathrm{c}$ \\
\hline $\mathrm{T}_{4}=16,65$ & (A) a & $\mathrm{T}_{5}=18,79$ & (A) $b$ & $\mathrm{~T}_{6}=22,00$ & (A) $\mathrm{c}$ & $\mathrm{T}_{4}=15,34$ & $(\mathrm{AB}) \mathrm{a}$ & $\mathrm{T}_{5}=19,01$ & (A) $b$ & $\mathrm{~T}_{6}=21,91$ & (A) $\mathrm{c}$ \\
\hline $\mathrm{T}_{7}=15,91$ & (B) $\mathrm{a}$ & $\mathrm{T}_{8}=18,55$ & (A) $\mathrm{b}$ & $\mathrm{T}_{9}=21,06$ & (A) $\mathrm{c}$ & $\mathrm{T}_{7}=15,85$ & (B) a & $\mathrm{T}_{8}=18,74$ & (A) $b$ & $\mathrm{~T}_{9}=21,88$ & (A) $\mathrm{c}$ \\
\hline $\mathrm{T}_{10}=16,23$ & $(\mathrm{AB}) \mathrm{a}$ & $\mathrm{T}_{11}=19,11$ & (A) $b$ & $\mathrm{~T}_{12}=21,67$ & (A) $\mathrm{c}$ & $\mathrm{T}_{10}=16,02$ & (B) a & $\mathrm{T}_{11}=19,24$ & (A) b & $\mathrm{T}_{12}=22,40$ & (A) $\mathrm{c}$ \\
\hline $\mathrm{T}_{13}=13,23$ & (C) $\mathrm{a}$ & $\mathrm{T}_{14}=14,16$ & (B) $\mathrm{a}$ & $\mathrm{T}_{15}=18,61$ & (B) $\mathrm{b}$ & $\mathrm{T}_{13}=13,31$ & (C) $\mathrm{a}$ & $\mathrm{T}_{14}=17,43$ & (A) $b$ & $\mathrm{~T}_{15}=19,48$ & (B) $\mathrm{b}$ \\
\hline \multicolumn{6}{|c|}{ Erro relativo - Terrenos planos } & \multicolumn{6}{|c|}{ Erro relativo - Terrenos inclinados } \\
\hline $\mathrm{T}_{1}=-1,88$ & (A) $\mathrm{a}$ & $\mathrm{T}_{2}=6,22$ & (A) $b$ & $\mathrm{~T}_{3}=5,15$ & (A) $b$ & $\mathrm{~T}_{1}=5,63$ & (A) $\mathrm{a}$ & $\mathrm{T}_{2}=5,05$ & (A) $\mathrm{a}$ & $\mathrm{T}_{3}=7,92$ & (A) a \\
\hline $\mathrm{T}_{4}=-2,23$ & (A) $\mathrm{a}$ & $\mathrm{T}_{5}=4,26$ & (A) $b$ & $\mathrm{~T}_{6}=4,74$ & (A) $b$ & $\mathrm{~T}_{4}=4,55$ & $(\mathrm{AB}) \mathrm{a}$ & $\mathrm{T}_{5}=2,38$ & (A) a & $\mathrm{T}_{6}=7,31$ & (A) a \\
\hline $\mathrm{T}_{7}=2,27$ & (B) a & $\mathrm{T}_{8}=5,48$ & (A) $b$ & $\mathrm{~T}_{9}=8,92$ & (A) $\mathrm{c}$ & $\mathrm{T}_{7}=1,24$ & $(\mathrm{BC}) \mathrm{a}$ & $\mathrm{T}_{8}=4,11$ & (A) $a b$ & $\mathrm{~T}_{9}=7,39$ & (A) $b$ \\
\hline $\mathrm{T}_{10}=0,35$ & $(\mathrm{AB}) \mathrm{a}$ & $\mathrm{T}_{11}=2,55$ & (A) $\mathrm{a}$ & $\mathrm{T}_{12}=6,19$ & (A) $b$ & $\mathrm{~T}_{10}=0,26$ & (C) $\mathrm{a}$ & $\mathrm{T}_{11}=1,64$ & (A) $a b$ & $\mathrm{~T}_{12}=5,32$ & (A) $\mathrm{b}$ \\
\hline $\mathrm{T}_{13}=18,71$ & (C) $\mathrm{a}$ & $\mathrm{T}_{14}=27,82$ & (B) $\mathrm{b}$ & $\mathrm{T}_{15}=19,33$ & (B) $\mathrm{b}$ & $\mathrm{T}_{13}=16,80$ & (D) $\mathrm{a}$ & $\mathrm{T}_{14}=11,12$ & (A) $\mathrm{a}$ & $\mathrm{T}_{15}=17,81$ & (B) $\mathrm{a}$ \\
\hline
\end{tabular}

Nota: Para as comparações, foi empregada a estatística $t$ para amostras independentes, a 5\% de probabilidade. As letras maiúsculas entre parênteses na vertical indicam a diferença entre métodos, e as letras minúsculas na horizontal indicam a diferença entre as classes em um mesmo método. Tratamentos com letras iguais não diferem entre si.

Quando se avalia o efeito da classe no desempenho dos métodos em terreno plano, pela observação da tabela 3, nota-se que a altura média diferiu em todas as classes para todos os métodos, com exceção do método sem treinamento. Esse resultado era esperado, na medida em que as classes foram previamente definidas para diferirem em altura e os métodos captaram essa diferenciação, com exceção do método sem treinamento, que mais uma vez oferece indicações do seu pior desempenho. Mas o resultado mais importante diz respeito à variável erro médio. Nesse caso, nota-se que houve uma tendência de melhor desempenho dos métodos na classe 1, não havendo, em geral, diferenciação entre as classes 2 e 3 (Tabela 3).

No terreno inclinado, foram obtidos resultados semelhantes aos encontrados para o terreno plano, considerando-se a variável altura média. Por outro lado, quando se avalia o erro médio, observa-se que os equipamentos digitais passaram a ter o mesmo desempenho entre as classes. Isso ocorreu porque o desempenho deles foi reduzido na classe 1, tornando-o igual às demais classes, reforçando o efeito negativo da declividade sobre esse tipo de aparelho.

É interessante ponderar também que a magnitude dos erros médios cometidos pelos diferentes métodos nas diferentes classes de altura (Tabela 3 ) manteve-se em patamares aceitáveis (excetuando-se o método sem treinamento), alcançando valores menores que $1 \%$ nas melhores situações e de no máximo 8,92\% na situação mais desfavorável. De acordo com Gobbo et al. (2007), os hipsômetros, em suas melhores condições de funcionamento, geram erros da ordem de 1 a $2 \%$, não sendo possível garantir resultados exatos em medições de árvores em pé, quer pela natureza do aparelho, quer pela condição do povoamento, em que, muitas vezes, não é possível visualizar o ápice ou a base da árvore com nitidez, a partir de um mesmo ponto. Por outro lado, admitindo que o processo de aplicação dos métodos possa passar por treinamentos mais intensos e novos aperfeiçoamentos, pode-se inferir que as estimações de altura total possam ser feitas com precisão consideravelmente elevada.

Avaliando os resultados da tabela 4, ao serem comparadas as alturas médias obtidas em cada tratamento com as alturas médias observadas correspondentes, podem-se corroborar alguns resultados encontrados na tabela 3. O método de estimação visual sem treinamento realmente apresenta o pior 
desempenho, ressaltando-se o fato de que esse método subestima de forma significativa a altura média em todas as classes, especialmente em terreno plano. Essa tendência em subestimar é em princípio algo difícil de explicar, mas pode estar associada ao receio do mensurador de estar exagerando na estimativa. Por outro lado, isso é muito subjetivo e pode variar de pessoa para pessoa, sendo um dos grandes inconvenientes desse método.

Outro resultado que confirma resultados anteriores é o pior desempenho dos aparelhos digitais no terreno inclinado, especialmente do Vertex. Para o modelo VertexIII utilizado neste trabalho, após sucessivos testes, foi constatado que ele não possibilita a correção da distância em terrenos com declividade, porém uma nova versão desse equipamento encontra-se disponível, podendo prover resultados melhores. Por outro lado, o método que emprega a régua como referência na estimação da altura parece ter sido favorecido no terreno inclinado. Essa melhora se deve à melhor possibilidade de visualização, pois, ao se deslocar para uma parte mais alta no terreno, em relação à árvore a ser estimada, é possível obter uma melhor estimação, visto que a copa da árvore estará mais visível ao mensurador. Já o método de estimação visual com treinamento parece não ter sido afetado pela inclinação do terreno.

Ainda com base na tabela 4, quando se avalia o efeito das classes na estimação da altura, nota-se que os aparelhos digitais parecem ser menos influenciados pelo aumento da altura, especialmente no terreno plano. Os métodos de estimação visual diferiram em todas as situações da altura real observada para a classe 3, tanto para o terreno plano quanto para inclinado, mostrando a dificuldade em se fazer estimação visual para as árvores mais altas, mesmo com treinamento ou tendo uma referência como auxílio.

Tabela 4. Médias de cada tratamento com a respectiva média da altura real observada em cada uma das classes, para terrenos planos e inclinados.

Table 4. Means of each treatment with respective average real height observed in each class, for flat and sloping ground.

\begin{tabular}{|c|c|c|c|c|c|c|}
\hline \multirow[b]{2}{*}{ Tratamento } & \multicolumn{3}{|c|}{ Terreno plano } & \multicolumn{3}{|c|}{ Terreno inclinado } \\
\hline & Média & $\begin{array}{l}\text { Desvio } \\
\text { Padrão } \\
\end{array}$ & Significância & Média & $\begin{array}{l}\text { Desvio } \\
\text { Padrão } \\
\end{array}$ & Significância \\
\hline 1 & 16,62 & 1,8351 & ns & 15,15 & 1,6979 & $*$ \\
\hline 2 & 18,38 & 2,2662 & $*$ & 18,53 & 1,8835 & $*$ \\
\hline 3 & 21,93 & 3,2412 & ns & 21,77 & 2,8420 & $*$ \\
\hline 4 & 16,65 & 2,0688 & $\mathrm{~ns}$ & 15,34 & 2,0266 & $*$ \\
\hline 5 & 18,79 & 2,7011 & $\mathrm{~ns}$ & 19,01 & 3,1855 & $\mathrm{~ns}$ \\
\hline 6 & 22,00 & 3,1286 & $\mathrm{~ns}$ & 21,91 & 3,0219 & $*$ \\
\hline 7 & 15,91 & 0,9937 & $*$ & 15,85 & 0,8984 & $\mathrm{~ns}$ \\
\hline 8 & 18,55 & 1,5718 & $*$ & 18,74 & 1,8492 & $\mathrm{~ns}$ \\
\hline 9 & 21,06 & 2,1075 & $*$ & 21,88 & 2,0070 & $*$ \\
\hline 10 & 16,23 & 1,0369 & $\mathrm{~ns}$ & 16,02 & 0,9797 & $\mathrm{~ns}$ \\
\hline 11 & 19,11 & 1,4282 & $\mathrm{~ns}$ & 19,24 & 1,8702 & $\mathrm{~ns}$ \\
\hline 12 & 21,67 & 1,9955 & $*$ & 22,40 & 1,9217 & $*$ \\
\hline 13 & 13,23 & 2,9194 & $*$ & 13,31 & 1,9004 & $*$ \\
\hline 14 & 14,16 & 3,8951 & $*$ & 17,43 & 4,6105 & $\mathrm{~ns}$ \\
\hline 15 & 18,61 & 5,8815 & $*$ & 19,48 & 4,2860 & $*$ \\
\hline Classe 1 Real & 16,30 & - & - & 16,07 & - & - \\
\hline Classe 2 Real & 19,62 & - & - & 19,55 & - & - \\
\hline Classe 3 Real & 23,13 & - & - & 23,70 & - & - \\
\hline
\end{tabular}

Nota: Considerando-se a estatística $t$ para amostras independentes, a 5\% de probabilidade: *: significativo a 5\% de probabilidade pelo teste $t{ }^{\text {ns: }}$ : não significativo a $5 \%$ de probabilidade pelo teste $t$.

Análise gráfica dos resíduos

De acordo com a figura 1, onde é apresentada uma análise gráfica dos resíduos em terrenos planos, nota-se, para a classe 1 de altura, que os métodos de estimativa com hipsômetro Vertex, clinômetro digital, estimativa visual com auxílio da régua de 15 metros e estimativa visual com treinamento apresentaram distribuição residual não tendenciosa. 
Classe 1
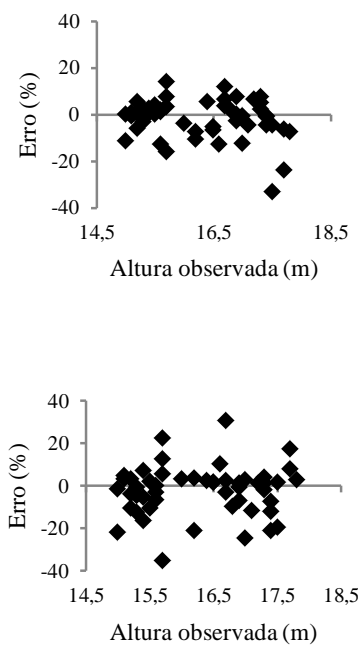

Classe 2

Método 1 - Estimação com hipsômetro Vertex
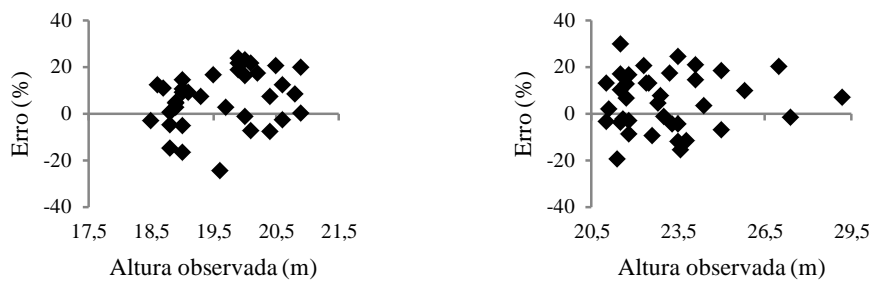

Método 2 - Estimação com clinômetro Digital

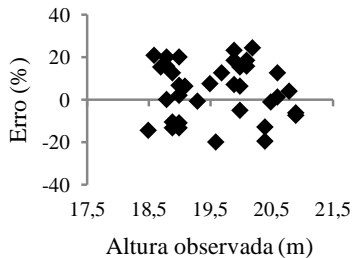

Classe 3

Método 3 - Estimação visual com auxílio de uma régua graduada de 15 metros
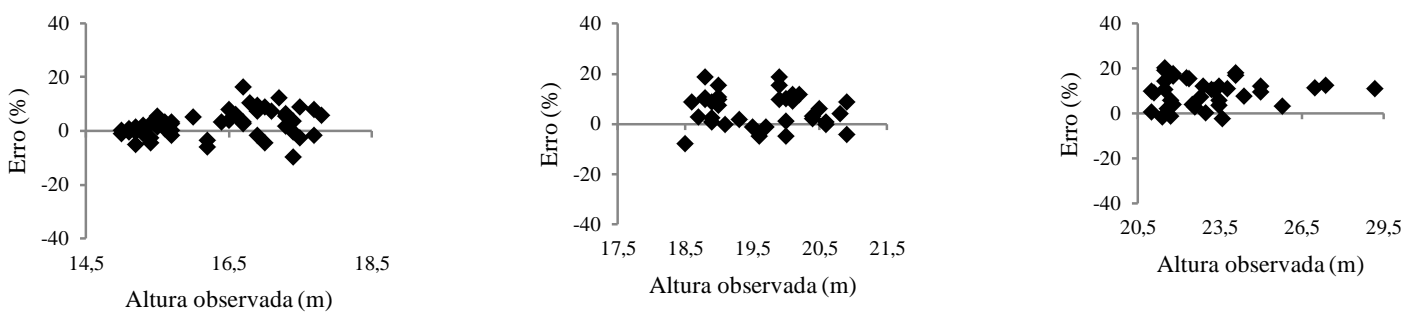

Método 4 - Estimação visual com treinamento
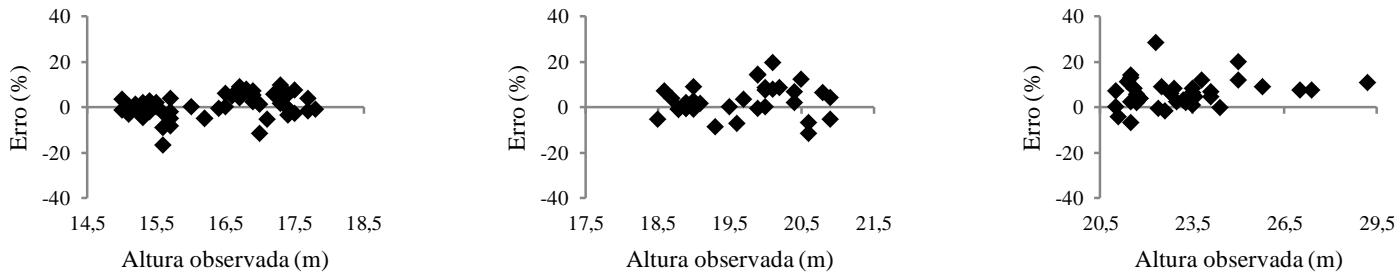

Método 5 - Estimação visual sem treinamento
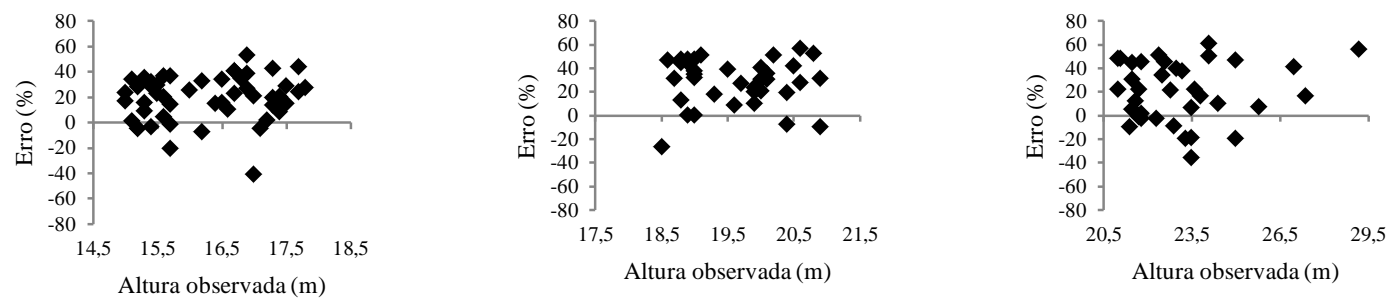

Figura 1. Distribuição dos resíduos de altura em percentagem, em terreno plano, para os cinco métodos avaliados nas classes de altura 1 (15-17,99 m), 2 (18-20,99 m) e 3 (>21 m), Floresta do Rosal.

Figure1. Residual distribution of height in percentage, on flat terrain for five methods evaluated in height classes 1 (15-17.99 m), 2 (18-20.99 m) and 3 (>21 m), Rosal Forest, Guaçuí, ES. 
Para terrenos inclinados, de acordo com a figura 2, os métodos de estimativa com hipsômetro Vertex e clinômetro digital apresentaram uma leve tendência a subestimar a altura. Já os métodos de estimativa visual com auxílio da régua e com treinamento apresentaram distribuição residual não tendenciosa.

Para as duas condições de terreno consideradas, para o método de estimativa visual sem treinamento, observou-se uma tendência clara em subestimar a altura, sendo essa tendência, além da classe 1 , observada em todas as classes de altura avaliadas.

Para a classe 2 de altura, em terrenos planos, nota-se uma pequena tendência de subestimativa da altura pelos métodos de estimativa com hipsômetro Vertex e clinômetro digital. O método de estimativa com auxílio da régua mostrou uma tendência evidente em subestimar, e o método de estimativa visual com treinamento apresentou distribuição residual com pequena tendência de subestimação para as árvores com alturas maiores dentro da referida classe. Em terrenos inclinados, todos os métodos apresentaram uma leve tendência em subestimar a altura, sendo essa tendência bem mais evidente no método sem treinamento.

Ao considerar a classe 3, observou-se uma tendência mais clara de subestimativa das alturas estimadas para os cinco métodos avaliados nas duas condições de terreno.

Sendo assim, para as diferentes condições de terreno, a tendência em subestimar aumenta, à medida que aumentam as classes de altura, ou seja, os métodos avaliados apresentaram estimativas mais confiáveis para a menor classe de altura (Classe 1) e menos confiáveis para a maior classe de altura (Classe 3), com exceção para o método 5, que subestimou a altura das árvores em todas as classes.

Testes complementares de precisão dos métodos

$\mathrm{Na}$ tabela 5, estão apresentadas as estatísticas viés $(V)$, média das diferenças absolutas $(M D)$ e desvio padrão das diferenças $(D P D)$ para as estimativas de altura, em terreno plano e inclinado, pelos diferentes métodos nas classes 1, 2 e 3 . Os valores positivos e negativos da estatística $V$ indicam subestimativa e superestimativa, respectivamente. Os menores valores das três estatísticas testadas indicam que o método de estimação de altura apresenta maior precisão.

A tabela 5 contém também as notas atribuídas para as estimativas de altura referentes às classes 1,2 e 3, baseadas nas estatísticas da mesma. No caso da estatística viés, a menor nota foi atribuída para a classe que apresentou resultado mais próximo de zero (0), independentemente de ser positivo ou negativo.

Para as duas situações de terreno, verifica-se que, para a classe 1 de altura, o método 4 apresentou o melhor resultado, seguido pelos métodos $3,1,2$ e 5 , nessa ordem. O pior desempenho dos métodos 1 e 2, quando comparados aos métodos 3 e 4, pode estar relacionado à falta de habilidade do operador, cansaço em detrimento da monotonia da atividade, calibração do instrumento, tempo de coleta e boa visibilidade do topo e da base da árvore, conforme discutido por Scolforo e Figueiredo Filho (1998).

Para a avaliação da altura pelos métodos 3 e 4, os mensuradores conseguiam visualizar melhor a copa das árvores, pois, no caso de dificuldade em vê-la a certa distância, o mensurador podia se aproximar, conseguindo ainda assim realizar a estimação. Já no caso dos métodos 1 e 2, ao se posicionar a pequenas distâncias da árvore, o ângulo de inclinação dos equipamentos (Vertex e clinômetro digital) em relação à copa era aumentado, fazendo com que pequenas oscilações no momento da leitura correspondessem a erros mais significativos.

O método 5 se mostrou pouco eficiente para estimação de altura em todas as classes avaliadas, o que era esperado, visto que a aplicação desse método não possui um fundamento científico para o seu desenvolvimento. Nas classes 2 e 3, que correspondem às árvores de maior altura, os resultados foram ainda menos precisos.

Para as três classes de altura, o método 4 apresentou melhor desempenho que o método 3 na estimação de altura. Considerando que o mesmo operador realizou as duas atividades, primeiro para o método 4 e após um período de tempo para o método 3, foi possível verificar que o treinamento obtido com a escalada foi perdido com o passar do tempo. Isso não quer dizer que o treinamento não funcione, mas que ele precisa ser intensificado, lembrando que as estimativas de altura total obtidas neste trabalho foram realizadas por mensuradores pouco experientes.

Ainda considerando a classe 2, entre os métodos 1 e 2 houve um empate em termos de precisão, tendo em vista as estatísticas consideradas, para as duas condições de terreno, ocorrendo o mesmo na classe 3. Já para terrenos planos, houve uma melhora na precisão do método 2 com relação ao método 1 . Assim, o método 1 não perdeu a precisão tão rapidamente com o aumento das classes de altura das árvores em terrenos inclinados, mas em terrenos planos a precisão do método 1 caiu de maneira mais evidente. 
Classe 2

Método 1 - Estimação com hipsômetro Vertex
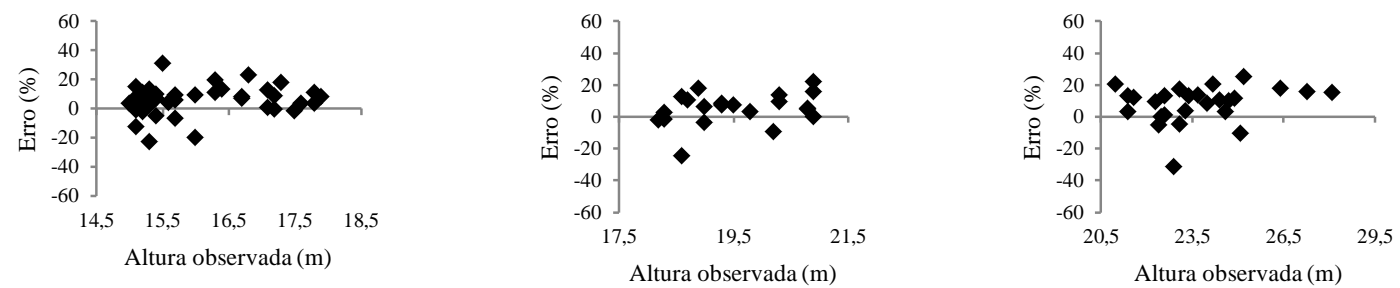

Método 2 - Estimação com clinômetro Digital
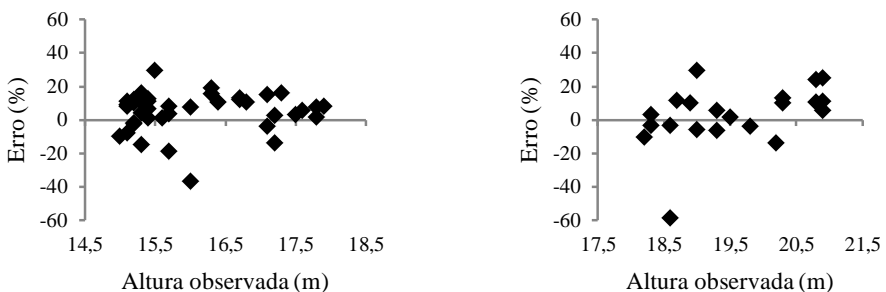

Método 3 - Estimação visual com auxílio de uma régua graduada de 15 metros
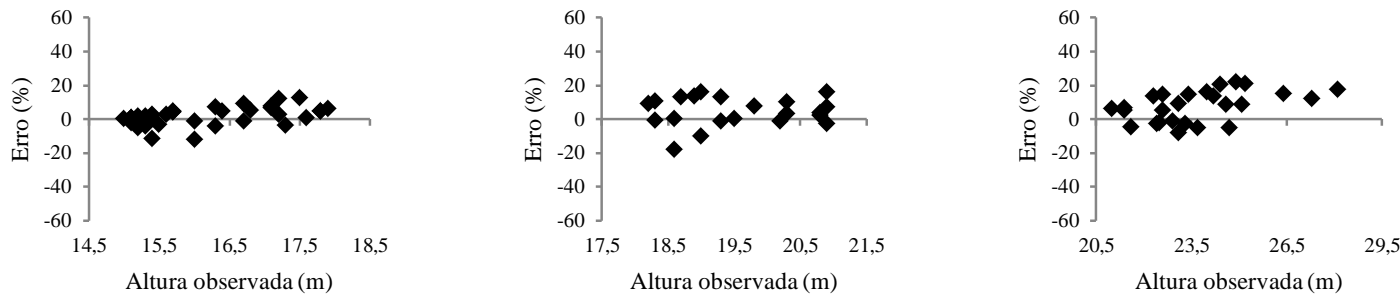

Método 4 - Estimação visual com treinamento
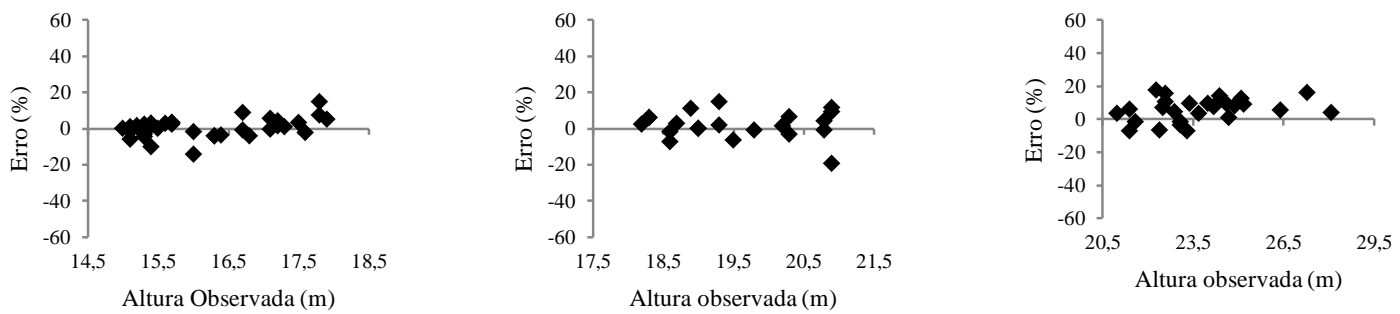

Método 5 - Estimação visual sem treinamento
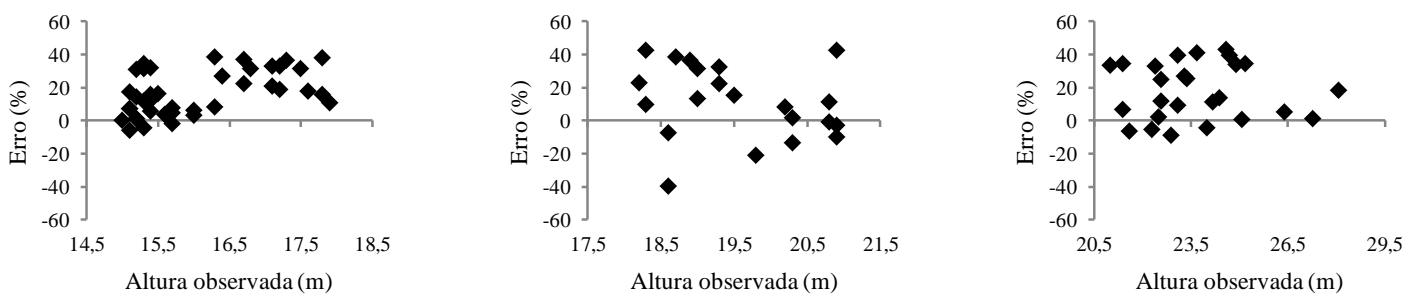

Figura 2. Distribuição dos resíduos de altura em percentagem, terreno inclinado, para os cinco métodos avaliados nas classes de altura 1 (15-17,99 m), 2 (18-20,99 m) e 3 (>21 m), Floresta do Rosal.

Figure 2. Residual distribution of height in percentage, on a slope ground, for five methods evaluated in height classes 1 (15-17.99 m), 2 (18-20.99 m) and 3 (>21m), Rosal Forest, Guaçuí, ES. 
Tabela 5. Estatísticas viés $(V)$, média das diferenças absolutas $(M D)$ e desvio padrão das diferenças $(D P D)$ para as estimativas de altura, em terreno plano e inclinado, referentes aos cinco métodos avaliados nas classes de altura 1, 2 e 3, com as respectivas notas atribuídas com base nas estatísticas.

Table 5. Statistics bias (V), mean absolute difference (MD) and standard deviations of differences (DPD) to estimate the height, inflat and sloping ground, for the five methods evaluated in height classes1, 2 and 3 with their scores assigned based on the statistics.

\begin{tabular}{|c|c|c|c|c|c|c|c|c|c|}
\hline Métodos & Classe & Tratamento & $V$ & $M D$ & $D P D$ & $\boldsymbol{V}$ & $M D$ & $D P D$ & Total \\
\hline \multicolumn{10}{|c|}{ Terrenos planos } \\
\hline \multirow[t]{4}{*}{1 - HipsômetroVertex } & 1 & 1 & $-0,32157$ & 0,98824 & 0,20104 & 2 & 3 & 3 & 8 \\
\hline & 2 & 2 & 1,24444 & 2,20556 & 0,39956 & 4 & 3 & 3 & 10 \\
\hline & 3 & 3 & 1,20270 & 2,55946 & 0,47063 & 2 & 4 & 3 & 9 \\
\hline & Total & - & & & & 8 & 10 & 9 & 27 \\
\hline \multirow[t]{4}{*}{2 - Clinômetro digital } & 1 & 4 & $-0,35686$ & 1,37255 & 0,27088 & 3 & 4 & 4 & 11 \\
\hline & 2 & 5 & 0,83056 & 2,29722 & 0,43229 & 2 & 4 & 4 & 10 \\
\hline & 3 & 6 & 1,12703 & 2,28919 & 0,47953 & 1 & 3 & 4 & 8 \\
\hline & Total & - & & & & 6 & 11 & 12 & 29 \\
\hline \multirow[t]{4}{*}{3 - Auxílio da régua de $15 \mathrm{~m}$} & 1 & 7 & 0,38431 & 0,70196 & 0,11881 & 4 & 2 & 1 & 7 \\
\hline & 2 & 8 & 1,06944 & 1,35833 & 0,22072 & 3 & 2 & 1 & 6 \\
\hline & 3 & 9 & 2,07297 & 2,14324 & 0,23914 & 4 & 2 & 1 & 7 \\
\hline & Total & - & & & & 11 & 6 & 3 & 20 \\
\hline \multirow[t]{4}{*}{4 - Com treinamento } & 1 & 10 & 0,07059 & 0,65490 & 0,12271 & 1 & 1 & 2 & 4 \\
\hline & 2 & 11 & 0,50833 & 1,06944 & 0,22980 & 1 & 2 & 2 & 5 \\
\hline & 3 & 12 & 1,45946 & 1,62162 & 0,25275 & 3 & 1 & 2 & 6 \\
\hline & Total & - & & & & 5 & 4 & 6 & 15 \\
\hline \multirow[t]{4}{*}{5 - Sem treinamento } & 1 & 13 & 3,07059 & 3,65882 & 0,41837 & 5 & 5 & 5 & 15 \\
\hline & 2 & 14 & 5,45833 & 5,94722 & 0,65342 & 5 & 5 & 5 & 15 \\
\hline & 3 & 15 & 4,52432 & 6,06486 & 0,99690 & 5 & 5 & 5 & 15 \\
\hline & Total & - & & & & 15 & 15 & 15 & 45 \\
\hline \multicolumn{10}{|c|}{ Terrenos inclinados } \\
\hline \multirow[t]{5}{*}{1 - Hipsômetro Vertex } & 1 & 1 & 0,92063 & 1,49023 & 0,25901 & 4 & 3 & 3 & 10 \\
\hline & 2 & 2 & 1,01766 & 1,76702 & 0,44270 & 4 & 3 & 3 & 10 \\
\hline & 3 & 3 & 1,93446 & 2,85598 & 0,56069 & 4 & 3 & 3 & 10 \\
\hline & Total & - & & & & 12 & 9 & 9 & 30 \\
\hline & 1 & 4 & 0,73750 & 1,62250 & 0,29989 & 3 & 4 & 4 & 11 \\
\hline \multirow[t]{3}{*}{2 - Clinômetro digital } & 2 & 5 & 0,53810 & 2,48095 & 0,78261 & 2 & 4 & 4 & 10 \\
\hline & 3 & 6 & 1,79231 & 3,04615 & 0,62089 & 2 & 4 & 4 & 10 \\
\hline & Total & - & & & & 7 & 12 & 12 & 31 \\
\hline \multirow[t]{4}{*}{3 - Auxílio da régua de $15 \mathrm{~m}$} & 1 & 7 & 0,22250 & 0,65750 & 0,13772 & 2 & 2 & 2 & 6 \\
\hline & 2 & 8 & 0,80952 & 1,45714 & 0,37389 & 3 & 2 & 2 & 7 \\
\hline & 3 & 9 & 1,82308 & 2,42308 & 0,45075 & 3 & 2 & 2 & 7 \\
\hline & Total & - & & & & 8 & 6 & 6 & 20 \\
\hline \multirow[t]{4}{*}{4 - Com treinamento } & 1 & 10 & 0,05750 & 0,55750 & 0,12968 & 1 & 1 & 1 & 3 \\
\hline & 2 & 11 & 0,31429 & 1,10476 & 0,33921 & 1 & 1 & 1 & 3 \\
\hline & 3 & 12 & 1,30000 & 1,80769 & 0,33638 & 1 & 1 & 1 & 3 \\
\hline & Total & - & & & & 3 & 3 & 3 & 9 \\
\hline \multirow[t]{4}{*}{5 - Sem treinamento } & 1 & 13 & 2,76000 & 2,85500 & 0,35941 & 5 & 5 & 5 & 15 \\
\hline & 2 & 14 & 2,12381 & 3,89524 & 0,96153 & 5 & 5 & 5 & 15 \\
\hline & 3 & 15 & 4,21923 & 4,65769 & 0,80024 & 5 & 5 & 5 & 15 \\
\hline & Total & - & & & & 15 & 15 & 15 & 45 \\
\hline
\end{tabular}

De qualquer forma, ao serem avaliadas todas as classes de altura conjuntamente, o método 1 , em geral,apresentou melhor precisão quando comparado ao método 2, para as duas condições de terreno. 
É evidente que, apesar da utilização de instrumentos óticos de alta precisão, como é o caso do Vertex, torna-se necessário dispor de pessoas que possam estimar visualmente a altura das árvores com razoável precisão. Segundo Bechtold et al. (1998), pode-se esperar um aumento no nível de precisão da estimativa ocular, desde que as pessoas responsáveis pelas estimativas sejam treinadas de forma mais intensiva e realizem uma "calibração" em cada parcela a ser inventariada, por meio da medição prévia de algumas árvores com aparelhos de estimação de altura.

\section{Análise do tempo médio para os métodos aplicados}

A tabela 6 apresenta os testes de médias para o tempo demandado pelos tratamentos avaliados, em terreno plano e inclinado, considerando-se a estatística $t$ para amostras independentes, a $5 \%$ de probabilidade. As seguintes hipóteses estatísticas foram avaliadas:

Hipótese para classe 1) $\mathrm{T}_{1}=\mathrm{T}_{4}=\mathrm{T}_{7}=\mathrm{T}_{10}=\mathrm{T}_{13}$

Hipótese para classe 2) $\mathrm{T}_{2}=\mathrm{T}_{5}=\mathrm{T}_{8}=\mathrm{T}_{11}=\mathrm{T}_{14}$

Hipótese para classe 3) $\mathrm{T}_{3}=\mathrm{T}_{6}=\mathrm{T}_{9}=\mathrm{T}_{12}=\mathrm{T}_{15}$

Com base na tabela 6, tanto para o terreno plano quanto para o inclinado, nota-se uma tendência geral dos métodos de estimação visual de consumirem menos tempo, com exceção do método que empregou o uso da régua. Esse resultado era esperado, uma vez que a operação consiste apenas em localizar a melhor posição e estimar a altura sem o uso de qualquer aparelho.

Os aparelhos digitais apresentaram um maior consumo de tempo, como era de se esperar, ressaltando que o Vertex apresentou melhor desempenho que o clinômetro, em função de que, para o Vertex, não há necessidade de medidas de distância com trena. O método de estimativa com auxílio de uma régua foi o que apresentou o maior consumo de tempo, pois exige alto esforço físico, que, ao longo do dia, acumula cansaço para o operador, diminuindo assim o rendimento da atividade. Exceções a essa tendência geral foram encontradas na classe $2 \mathrm{em}$ terreno plano, em que o clinômetro e o Vertex tiveram desempenho igual. Isso pode ter tido uma causa aleatória, não sendo algo esperado. Outra exceção ocorreu na classe $3 \mathrm{em}$ terreno inclinado, em que o clinômetro teve o mesmo desempenho de tempo que o método visual com auxílio da régua. Esse resultado provavelmente ocorreu pela dificuldade em corrigir o efeito da declividade para realizar a estimativa, combinado com a dificuldade de estimar árvores mais altas.

Tabela 6. Comparação do tempo médio entre os tratamentos avaliados nas diferentes classes, tal como definido na tabela 1 , em terreno plano e inclinado.

Table 6. Comparison of average time among treatments evaluated in different classes as defined in table 1 , flat and sloped ground.

\begin{tabular}{|c|c|c|c|c|c|}
\hline \multicolumn{6}{|c|}{ Estimativa de tempo para estimação da altura das árvores - Terreno plano } \\
\hline $\mathrm{T} 1=91,45$ & (A) a & $\mathrm{T} 2=100,11$ & (A) a & $\mathrm{T} 3=103,97$ & (A) a \\
\hline $\mathrm{T} 4=118,55$ & (B) a & T5 $=117,89$ & (A) ab & $\mathrm{T} 6=133,54$ & (B) $\mathrm{b}$ \\
\hline $\mathrm{T} 7=163,63$ & (C) a & $\mathrm{T} 8=158,00$ & (B) a & $\mathrm{T} 9=158,24$ & (C) a \\
\hline $\mathrm{T} 10=33,53$ & (D) a & $\mathrm{T} 11=33,50$ & (C) a & $\mathrm{T} 12=34,97$ & (D) $\mathrm{a}$ \\
\hline $\mathrm{T} 13=8,20$ & (E) a & $\mathrm{T} 14=9,81$ & (D) $\mathrm{a}$ & $\mathrm{T} 15=7,73$ & (E) a \\
\hline \multicolumn{6}{|c|}{ Estimativa de tempo para estimação da altura das árvores - Terreno inclinado } \\
\hline $\mathrm{T} 1=95,13$ & (A) a & $\mathrm{T} 2=105,48$ & (A) b & $\mathrm{T} 3=111,58$ & (A) $\mathrm{b}$ \\
\hline $\mathrm{T} 4=127,70$ & (B) $\mathrm{a}$ & $\mathrm{T} 5=137,86$ & (B) $a b$ & $\mathrm{~T} 6=152,38$ & (B) $\mathrm{b}$ \\
\hline $\mathrm{T} 7=151,28$ & (C) a & $\mathrm{T} 8=165,95$ & (C) $\mathrm{a}$ & $\mathrm{T} 9=162,92$ & (B) $\mathrm{a}$ \\
\hline $\mathrm{T} 10=33,68$ & (D) a & $\mathrm{T} 11=25,71$ & (D) a & $\mathrm{T} 12=36,96$ & (C) $\mathrm{a}$ \\
\hline $\mathrm{T} 13=6,38$ & (E) a & $\mathrm{T} 14=12,86$ & (E) $a b$ & $\mathrm{~T} 15=8,15$ & (D) $\mathrm{b}$ \\
\hline
\end{tabular}

Nota: Para as comparações, foi empregada a estatística $t$ para amostras independentes, a $5 \%$ de probabilidade. As letras maiúsculas entre parênteses na vertical indicam a diferença entre métodos, e as letras minúsculas na horizontal indicam a diferença entre as classes em um mesmo método. Tratamentos com letras iguais não diferem entre si.

Quando se avalia o efeito da classe no tempo médio para estimar a altura total em terreno plano, pela observação da tabela 6 , nota-se que o tempo médio foi igual em todas as classes para todos os 
métodos, com exceção do método de estimação com clinômetro digital, que, com o aumento da classe, demandou maior tempo para estimar a altura, podendo isso estar relacionado à necessidade da medida de distância com a trena, para a qual, nas classes mais altas, há necessidade de um maior afastamento da árvore, a fim de se obter um melhor ponto de enquadramento.

No terreno inclinado, os métodos com treinamento e o que empregou a régua não tiveram seus desempenhos alterados ao longo das classes, isto é, não houve efeito de classe no terreno inclinado, assim como ocorreu no terreno plano. Por outro lado, com o aparelho Vertex, no terreno inclinado, diferentemente do que ocorreu no terreno plano, consumiu-se mais tempo nas classes maiores, denotando que a declividade afetou o desempenho desse aparelho. Já o clinômetro manteve a tendência de consumir mais tempo para classes maiores no terreno inclinado. $\mathrm{O}$ resultado inesperado foi o maior consumo de tempo para o método sem treinamento na segunda classe no terreno inclinado, o que também pode ter ocorrido por um efeito aleatório.

\section{CONCLUSÕES}

- O método de estimar a altura da árvore sem treinamento apresentou o pior desempenho quanto à precisão, nas duas condições de terreno avaliadas. Já o melhor desempenho foi para a estimativa com treinamento. A declividade afetou negativamente o desempenho dos equipamentos digitais, especialmente do Vertex, e o método com auxílio da régua favoreceu a estimação no terreno inclinado.

- A tendência em subestimar a altura das árvores aumenta à medida que aumentam as classes, com exceção para o método de estimação visual sem treinamento, que subestimou a altura em todas as classes, mostrando dificuldade para se fazer estimação visual para as árvores mais altas.

- Para as duas condições de terreno, nota-se tendência geral dos métodos de estimação visual de consumir menos tempo, com exceção do método que empregou o uso da régua, que consumiu o maior tempo médio em relação aos outros quatro. O Vertex teve melhor desempenho que o clinômetro.

- Quando se avaliou o efeito da classe no tempo médio para estimar a altura em terreno plano, notou-se que ele foi igual em todas as classes para todos os métodos, com exceção do método de estimação com clinômetro digital. Já em terreno inclinado, foram os métodos com treinamento e o que empregou a régua que não tiveram seus desempenhos alterados ao longo das classes.

\section{REFERÊNCIAS}

BECHTOLD, W. A.; ZARNOCH, S. J.; BURKMAN, W. G. Comparisons of modeled height predictions to ocular height estimates. Southern Journal of Applied Forestry, v. 22, n. 4, 1998.

CAMPOS, J. C. C.; LEITE, H. G. Mensuração florestal: perguntas e respostas. 3. ed. Viçosa, MG: UFV, 548 p., 2009.

GOBBO, E. P.; GORENSTEIN, M. R.; GOMES, J. E. Inventário dos povoamentos florestais da Estação Experimental de Tupi - Instituto Florestal - SP. Revista Científica Eletrônica de Engenharia Florestal. Publicação Científica da Faculdade de Agronomia e Engenharia Florestal de Garça/FAEF. Ano V, n. 9, 2007.

HUSCH, B.; MILLER, C. I.; BEERS, T. W. Forest mensuration. 3 ed. Malabar: Krieger Publishing Company, 402 p., 1993.

SCOLFORO, J. R. S.; FIGUEIREDO FILHO, A. Biometria Florestal: medição e volumetria de árvores. Lavras: UFLA/FAEPE, 310 p., 1998.

SOARES, C. P. B.; NETO, F. P.; SOUZA, A. L. Dendrometria e inventário florestal. Viçosa, MG: UFV, 276 p., 2006. 